\title{
The evolution of malignant and reactive $\gamma \delta+T$ cell clones in a relapse T-ALL case after allogeneic stem cell transplantation
}

\author{
Shaohua Chen ${ }^{1,2}$, Xin Huang ${ }^{3}$, Haitao Zheng ${ }^{2}$, Suxia Geng ${ }^{3}$, Xiuli Wu ${ }^{1,2}$, Lijian Yang $^{2}$, Jianyu Weng ${ }^{3}$, Xin Du ${ }^{3 *}$ \\ and Yangqiu $\mathrm{Li}^{1,2^{*}}$
}

\begin{abstract}
Background: To improve the outcome of patients with T-cell acute lymphoblastic leukemia (T-ALL), characterization of the biological features of T-ALL blast cells and the immune status of patients with T-ALL is needed to identify specific therapeutic strategies.

Findings: Using a novel approach based on the combination of fine-tiling comparative genomic hybridization (FT-CGH) and ligation-mediated PCR (LM-PCR), we molecularly identified a malignant $\gamma \delta+T$ cell clone with a

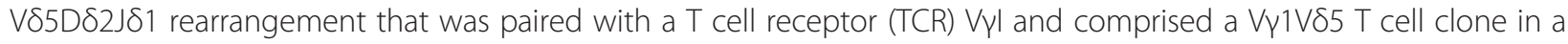
relapse T-ALL patient. This malignant V $\delta 5 T$ cell clone disappeared after chemotherapy, but the clone was detected again when disease relapsed post allogeneic hematopoietic stem cell transplantation (allo-HSCT) at 100 weeks. Using PCR and GeneScan analyses, the distribution and clonality of the TCR $V_{Y}$ and $V \delta$ subfamilies were examined before and after allo-HSCT in the patient. A reactive T cell clone with a $V \delta 4 D \delta 3 J \delta 1$ rearrangement was identified in all samples taken at different time points (i.e., 4, 8, 68, 100 and 108 weeks after allo-HSCT). The expression of this V $84+T$ cell clone was higher in the patient during complete remission (CR) post allo-HSCT and at disease relapse.
\end{abstract}

Conclusions: This study established a sensitive methodology to detect T cell subclones, which may be used to monitor minimal residual disease and immune reconstitution.

Keywords: $\gamma \delta$ T cell clone, T-ALL, FT-CGH, TCR, Allo-HSCT

\section{Findings}

T-cell acute lymphoblastic leukemia (T-ALL) comprises $25 \%$ of adult ALL cases, and its outcome is poorly understood. Among patients with T-ALL, approximately $40 \%$ achieve long-term remission [1-3]. Allogeneic hematopoietic stem cell transplantation (allo-HSCT) remains one of the best options for curing T-ALL. However, many patients cannot find an HLA-matched donor; therefore, haploidentical/mismatched HSCTs may be an alternative treatment for T-ALL $[4,5]$. The high T-ALL failure rate is mainly the result of an insufficient understanding of T-ALL biology, which hampers the

\footnotetext{
*Correspondence: miyadu@hotmail.com; yangqiuli@hotmail.com ${ }^{3}$ Department of Hematology, Guangdong General Hospital (Guangdong Academy of Medical Sciences), Guangzhou 510080, China 'Key Laboratory for Regenerative Medicine of Ministry of Education, Jinan University, Guangzhou 510632, China

Full list of author information is available at the end of the article
}

identification of reliable prognostic factors that enable appropriate therapy adjustment [1]. To improve T-ALL outcome, characterization of the biological features of T-ALL blast cells and the immune status of patients is needed to design specific therapeutic strategies [6-10]. T-ALL is generally considered to be a clonal disorder that arises from the expansion of committed lymphoid precursors, and leukemic clones in different patients vary due to the $\mathrm{T}$ cell receptor (TCR) gene rearrangements that occur during T-cell differentiation $[1,11]$. Moreover, TCR rearrangements also provide different recombination breakpoints that lead to the creation of fusion genes [12]. TCR rearrangement analysis may be used to determine T-ALL immunogenetic characteristics, and TCR rearrangements may be characterized by leukemia antigen-reactive $\mathrm{T}$ cell clones, which are thought to be specific to anti-leukemic cytotoxic T cells $[13,14]$.

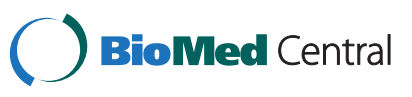


Table 1 Clinical therapy details for the patient with relapse T-ALL

\begin{tabular}{|c|c|c|c|c|c|}
\hline \multirow[t]{2}{*}{ Therapy date } & \multirow[t]{2}{*}{ Therapy } & \multirow[t]{2}{*}{ Response } & $\begin{array}{l}\text { Blast cells }(\%) \\
\text { post-treatment }\end{array}$ & \multirow[t]{2}{*}{ CSF } & \multirow[t]{2}{*}{ Intrathecal chemotherapy } \\
\hline & & & $\mathrm{BM} / \mathrm{PB}$ & & \\
\hline $18.11-01.12 .2009$ & $C T X, V C R, A D M, D X M$ & PR & $11 / 3$ & - & MTX + DXM \\
\hline 18.12-22.12.2009 & MTX, Ara-c, DXM & NR & $37 / 3$ & & \\
\hline 25.01-30.01.2010 & CTX, Ara-c, TPT & $N R$ & $47 / 1$ & - & Ara-c + DXM \\
\hline 05.03.2010 & $\begin{array}{c}\text { Allo-HSCT } \\
\text { (conditioning regimen: Flu, BU/CY) }\end{array}$ & $C R$ & $1 / 0$ & - & MTX + DXM \\
\hline 13.04 .2010 & CsA, MP & GVHD (Grade II) & $0.5 / 0$ & & \\
\hline 13.05 .2010 & CsA, MP & $\begin{array}{l}\text { GVHD under } \\
\text { control }\end{array}$ & $1.5 / 0$ & - & MTX + DXM \\
\hline $\begin{array}{l}12.08 .2010- \\
07.01 .2011\end{array}$ & Radiotherapy & CNSL cured & $0 / 0$ & + & $($ Ara-c + MTX $+D X M) \times 8$ times \\
\hline $26.04-21.05 .2012$ & VDS, NVT, L-ASPDXM & Relapse, CR & $3 / 0$ & - & $\begin{array}{c}\text { MTX + DXM (03.05 2012) Ara-c + DXM } \\
(25.05 .2012)\end{array}$ \\
\hline 08.06-06.07.2012 & VCR, NVT, L-ASP, DXM, CTX & $C R$ & $1 / 0$ & - & \\
\hline
\end{tabular}

Notes: $A D M$ adramycin, Ara-c cytarabine, BM bone marrow, $B U$ busulfan, CNSL central nervous system leukemia, CSA cyclosporin, CSF cerebrospinal fluid, $C T X$ cyclophosphamide, $C R$ complete remission, $C Y$ cyclophosphamide, DXM dexamethasone, Flu fludarabine, $L-A S P I$-asparaginase, $M P$ methylprednisolone, MTX Methotrexate, NR minor remission, NVT mitoxantrone, $P R$ partial remission, $P B$ peripheral blood, TPT Topotecan, VCR vincristine, VDS Vindesine.

In this study, using a novel approach based on the combination of fine-tiling comparative genomic hybridization (FT-CGH) and ligation-mediated PCR (LM-PCR) [15], which combines PCR and the GeneScan techniques $[16,17]$, we molecularly characterized the malignant and reactive $\gamma \delta+$ T cell clones in a patient with T-ALL before and after relapse 100 weeks post allo-HSCT.

A 25-year-old male patient was diagnosed with relapse T-ALL in November 2009. The diagnosis was based on cytomorphology, immunohistochemistry and cytoimmunological analyses. He underwent salvage chemotherapies for the next 3 months. However, his response assessments were partial remission (PR), minor remission (NR), and NR. In March 2010, the patient received an HLA-identical sibling peripheral blood (PB) HSCT after a conditioning regimen in addition to cyclosporin (CsA) in combination with a short course of mycophenolate mofetil (MMF) and four doses of methotrexate (MTX) for graft versus host disease (GVHD) prophylaxis as previously described [18]. Lumbar puncture and intrathecal chemotherapy were performed as normal. Four weeks after transplantation, the patient was stricken with fever, diarrhea, and a rash, which is considered Grade II GVHD. Eight weeks after transplantation, the GVHD was controlled with methylprednisolone (MP) and CsA treatment. Central nervous system leukemia (CNSL) was found 20 weeks post transplantation, and intrathecal chemotherapy and radiotherapy of the head were then applied. The patient achieved complete remission (CR) in the bone marrow and was consistently normal upon cerebrospinal fluid examination at 40, 52, and 68 weeks after transplantation. However, relapse was discovered 100 weeks after transplantation, and he underwent chemotherapy over the next two months and achieved remission. The treatment process is summarized in Table 1, and the details of the regimens used are summarized in Additional file 1: Table S1. As of this reporting, the patient remains in follow-up. Blood samples were collected with informed consent when the patient was diagnosed with relapse T-ALL before transplantation and at 4, 8, 68, 100 and 108 weeks post transplantation (Table 2 and Additional file 1: Table S2). PB was collected in an EDTA-containing collection tube, and PB mononuclear cells (PBMCs) were separated using the FicollHypaque gradient centrifugation method. All procedures were conducted in accordance with the guidelines of the Medical Ethics committees of Guangdong General Hospital according the guidelines of the health bureau of Guangdong Province, China.

\section{Malignant T-ALL clone}

To characterize the cellular T-ALL features and the T cell clonality at different time points before and after alloHSCT and at relapse post allo-HSCT, which may identify

\section{Table 2 Clinical details of the collected samples}

\begin{tabular}{lll}
\hline No. & Diagnosis & Disease status \\
\hline A & Relapse & Relapse \\
B & Pre allo-HSCT & NR \\
C & 4 W post allo-HSCT & CR \\
D & $8 \mathrm{~W}$ post allo-HSCT & CR \\
E & $68 \mathrm{~W}$ post allo-HSCT & CR \\
F & $100 \mathrm{~W}$ & Relapse \\
G & $108 \mathrm{~W}$ & MRD \\
\hline
\end{tabular}

Notes: allo-HSCT: $C R$ complete remission, MTD minimal residual disease, NR minor remission. 


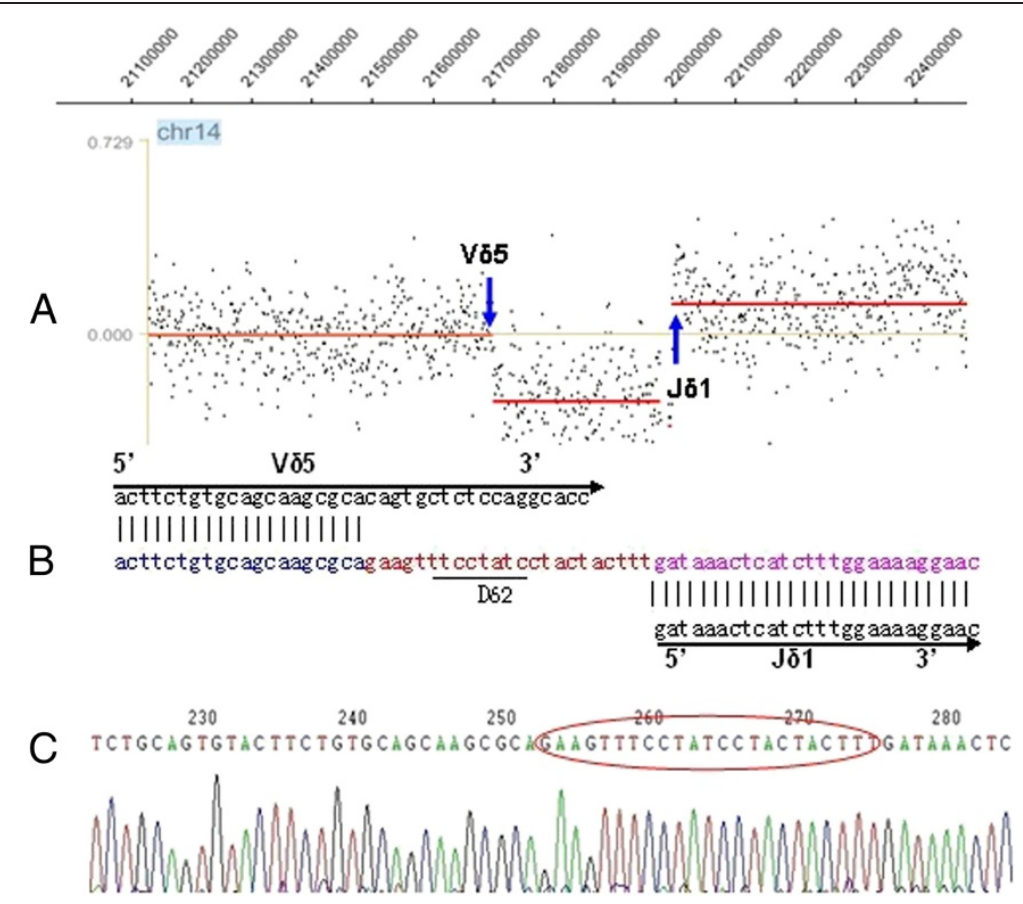

Figure 1 One TCR $\delta$ clone was identified by FT-CGH, LM-PCR and sequencing in a T-ALL case, and a V $\delta 5 D \delta 2 J \delta 1$ rearrangement was confirmed. A: FT-CGH analysis results for the TCR a $\delta$ locus in chromosome 14 with arrows indicating the breakpoints. B: Comparison of the GenBank sequences within the V $\delta 5, D \delta 2$, and J $\delta 1$ segments. C: Sequence of the V $\delta 5 D \delta 2 J \delta 1$ splice junction from a purified LM-PCR product. The sequences within the ellipse indicate the CDR3 segment including the $D \delta 2$ and $\mathrm{N}$ regions.

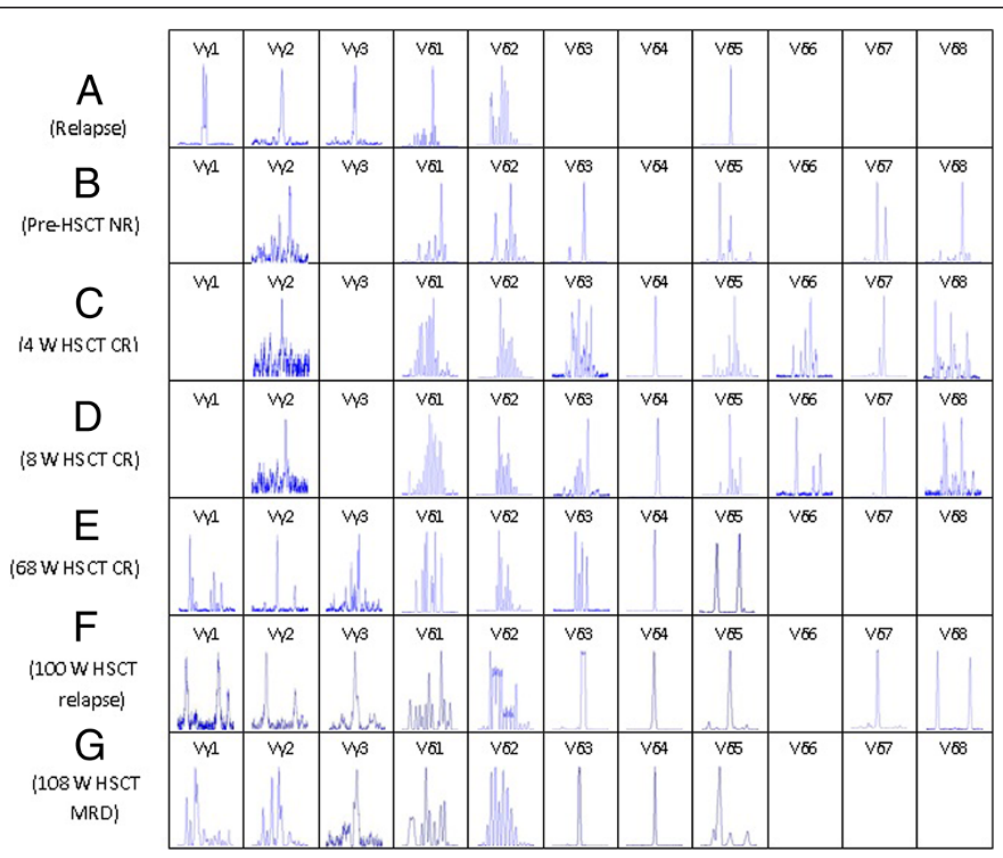

Figure 2 The distribution and clonality of the TCR $V_{Y}$ and $\mathbf{V} \delta$ subfamily $T$ cells in a patient with T-ALL at different time points before and after allo-HSCT. A: T-ALL relapse, B: pre-HSCT, C: 4 weeks post allo-HSCT, D: 8 weeks post allo-HSCT, E: 68 weeks post allo-HSCT, F: 100 weeks post allo-HSCT and disease relapse, and G: 108 weeks post allo-HSCT and after one cycle of chemotherapy. The monoclonal V85 subfamily could be found in samples $\mathbf{A}, \mathbf{F}$, and $\mathbf{G}$, and the oligoclonal $\vee \delta 4$ subfamily could be identified in samples $\mathbf{C}$ through $\mathbf{G}$. 
a factor associated with outcome, we analyzed the TCR breakpoint loci to identify chromosomal translocations and malignant $\mathrm{T}$ cell clones by the FT-CGH, LM-PCR, RT-PCT and GeneScan techniques [15-17].

FT-CGH using overlapping oligonucleotides designed to cover an entire genomic region of interest is a valuable tool for high-resolution chromosomal breakpoint characterization [15]. To achieve high resolution CGH $(<1 \mathrm{~kb})$, which is necessary for subsequent in vitro DNA amplification, a custom designed high-density, fine-tiling long oligonucleotide array consisting of 385,000 oligonucleotides $40-60 \mathrm{bp}$ in length was prepared using Maskless
Array Synthesizer (MAS) technology (NimbleGen Systems; Reykjavik, Iceland). This array, covering $24 \mathrm{Mb}$ of genome, was selected using the human genome browser hg18 assembly (University of California, Santa Cruz). The array included TCR $\alpha \delta$ and IgH loci, which are located on chromosome 14q11 (Chr14: 21,130 -22,130 kb) and 14q32 (Chr14: 105,080 -106,360 kb), respectively, and known to be frequently involved in chromosomal alterations in lymphoid malignancies. The neighboring oligonucleotides with an average distance of $63 \mathrm{bp}$ were grouped in 200, 400, and 1,000 bp clusters. After normalization with reference DNA from the HEK293 $\mathrm{T}$ cell line, the mean
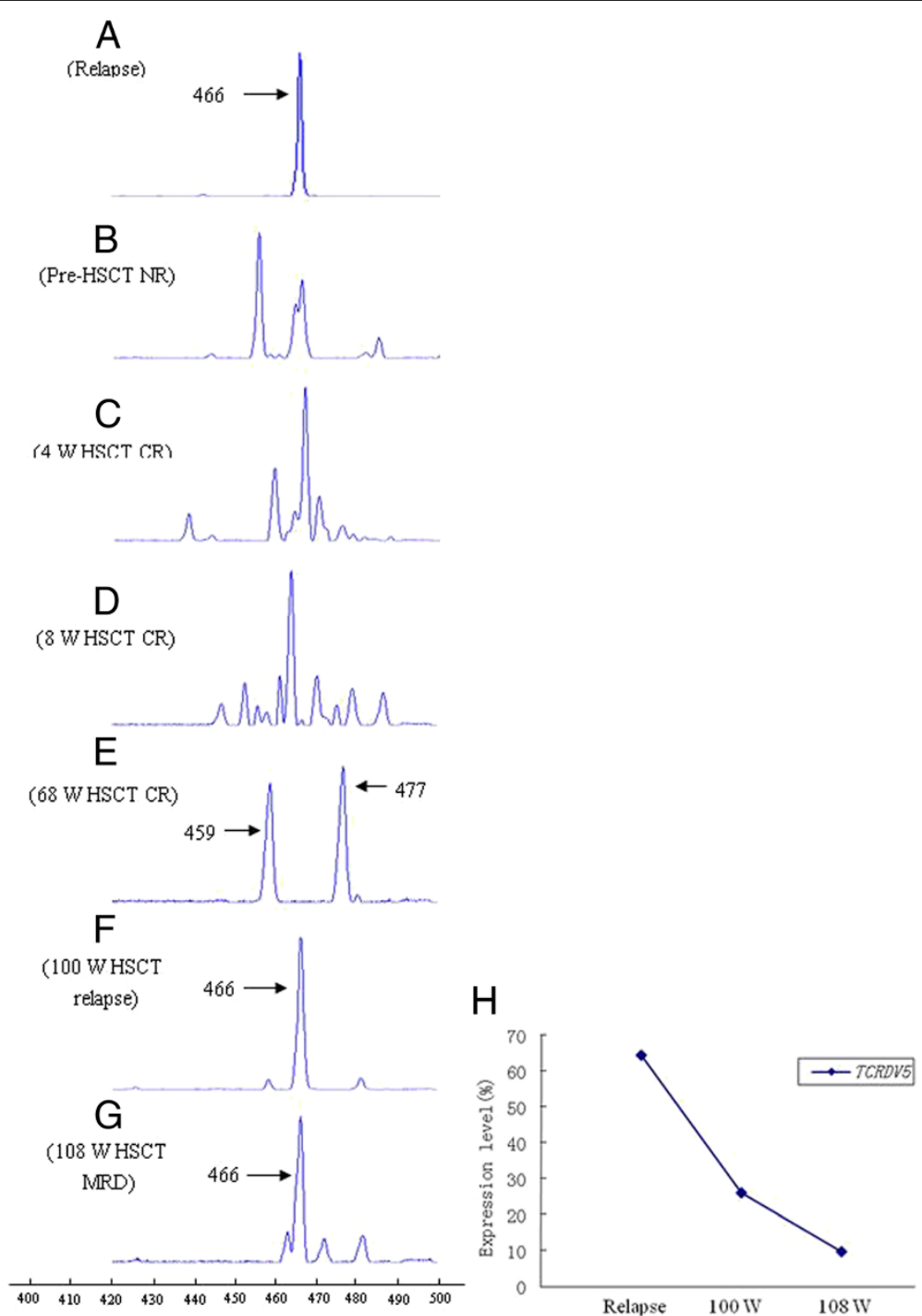

Figure 3 The clonality and size of V $\delta 5 T$ cells in a patient with T-ALL at different time points. V $\delta 5 T$ cell clonality was measured at different time points including the following: A: relapse before allo-HSCT (the monoclonal V $\delta 5$ product size was 466 bp), B: pre-HSCT, C: 4 weeks post allo-HSCT, D: 8 weeks post allo-HSCT, E: 68 weeks post allo-HSCT (the clonal V $\delta 5$ products were 459 and 477 bp, there were different from the malignant T cell clone with 466 bp), F: 100 weeks post allo-HSCT and disease relapse (a 466 bp monoclonal V $\delta 5$ product is again observed), and G: 108 weeks post allo-HSCT and after one chemotherapy cycle with minimal residual disease (MRD) remaining (a 466 bp monoclonal V $\delta 5$ product is still detectable). $\mathbf{H}$ : The expression level of the monoclonal the $\mathrm{V} \delta 5$ gene at different time points (i.e., relapse before allo-HSCT, relapse at 100 and 108 weeks post allo-HSCT and after one chemotherapy cycle with MRD remaining). 


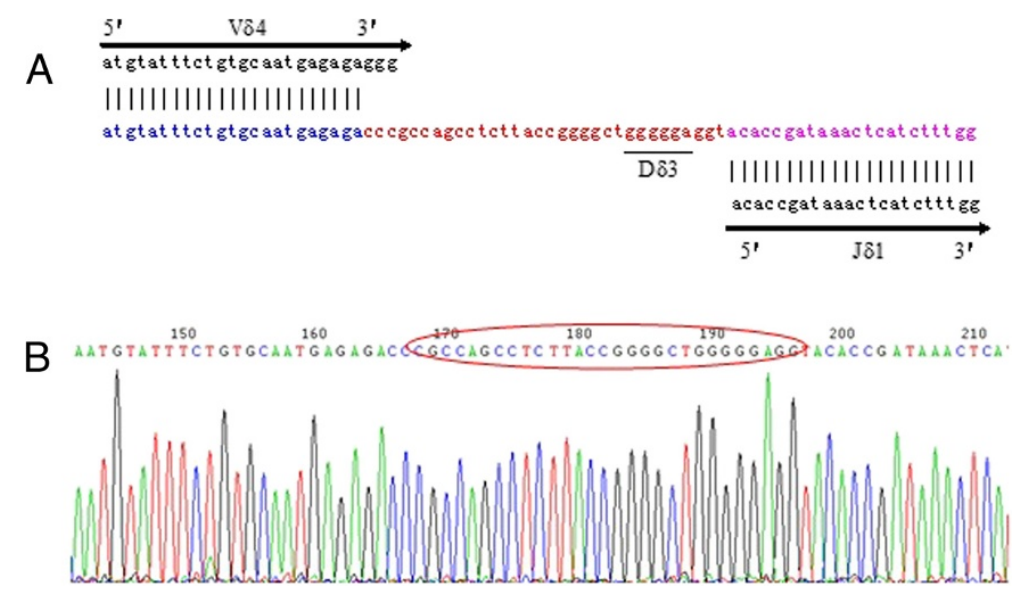

Figure 4 The CDR3 sequence in the V $\delta 4 D \delta 3 J \delta 1$ rearrangement. A: Sequence comparison of the V $\delta 4, D \delta 3$, and J $\delta 1$ segments from GenBank; B: sequence of the $V \delta 4 D \delta 3 J \delta 1$ splice junction.

fluorescence was analyzed using the SignalMap software (NimbleGen) [15].

FT-CGH analysis of the TCR $\alpha \delta$ locus (Chr 14: 21,130$22,130)$ from the T-ALL sample revealed breakpoints at the 21,700 kb (TCR V85) and 22,000 kb (TCR J81) loci (Figure 1A). LM-PCR using nested forward primers specific to the TCR V $\delta 5$ locus revealed a normal rearrangement i.e., a TCR V $\delta 5$ to $J \delta 1$ rearrangement. Direct sequencing of these LM-PCR products demonstrated the following details of the V $\delta 5 D \delta 2 J \delta 1$ rearrangement: the V $\delta 5$ breakpoint is located in chromosome 14 at position $21,701,606$ and the $J \delta 1$ breakpoint is located in chromosome 14 at position 21,988,926 (Figures $1 \mathrm{~B}$ and C). To confirm this TCR rearrangement, we used PCR to detect the $V \delta 5$ and $J \delta 1$ loci with specific primers and undigested genomic DNA. Thus, two 14q11.2 (TCR $\alpha \delta$ ) chromosome breakpoints were characterized as a TCR idiotype rearrangement. Therefore, it may be concluded that there were no additional breakpoints providing a recombination point for the TCR $\alpha \delta$ locus-related chromosomal translocation because the translocation of proto-oncogenes to TCR loci most likely occurred as the cell attempted to undergo $\mathrm{V}(\mathrm{D}) \mathrm{J}$ recombination, and it is likely that these translocations arise as a mistake in this process, depending on the location of the breakpoint in the receptor locus $[12,15,19,20]$. Using RT-PCR, GeneScan, and sequencing analysis, the V $\delta 5 D \delta 2 J \delta 1$ rearrangement was confirmed to be monoclonal (The information of the primers used for TCR V85-PCR was listed in Additional file 1: Table S3). Monoclonally expanded V $\delta 5 \mathrm{~T}$ cells, which were

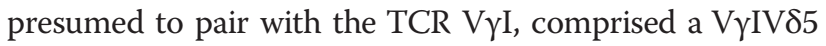
$\mathrm{T}$ cell clone (Figure 2A). Significantly, this malignant monoclone was lost after chemotherapy and allo-HSCT, but there was a gain in polyclonal V $\delta 5 \mathrm{~T}$ cells, which may pair with $\mathrm{V} \gamma \mathrm{II} \mathrm{T}$ cells and comprise many normal V $\gamma \mathrm{IIV} \delta 5$ $\mathrm{T}$ cell clones (Figure 2B) that arise from normal random $\mathrm{V}$
(D)J rearrangements [1]. Although the GeneScan results revealed biclonal V $\delta 5 \mathrm{~T}$ cells in the allo-HSCT sample taken after 68 weeks, these $\mathrm{T}$ cell clones were different from the malignant clone (Figure 3). The malignant monoclonal V85 product was 466 bp (Figure 3A), while both of the clonal V $\delta 5$ products from the post transplantation sample were 459 and 477 bp (Figure 3E). Moreover, the malignant monoclonal V $\delta 5$ clone reemerged 100 weeks after allo-HSCT treatment (Figure 3F), a time at which the patient underwent relapse, and the $\mathrm{V} \delta 5$ subfamily also included an oligoclone 108 weeks after allo-HSCT (Figure 3G), which was after chemotherapy and when the patient achieved remission. The CDR3 sequence of the V 85 clones at diagnosis and 100 and 108 weeks post allo-HSCT were determined, and the polyclonal and biclonal V85 T cell clones were found to contain different CDR3 sequences with different sizes. The V $\delta$ subfamily members preferentially expressed in $\mathrm{PB}$ from healthy and T-ALL clones include V $\delta 1$ and V $\delta 2$, while the V $\delta 5$ clone was reported to be rare $[21,22]$. Thus, this malignant $\mathrm{T}$ cell clone may serve as a biomarker for the detection of

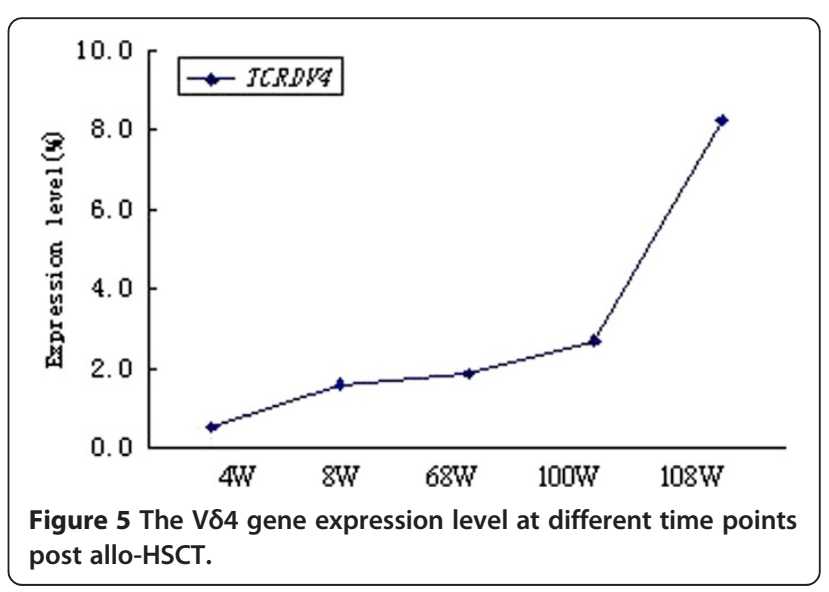


minimal residual disease. Indeed, a malignant V 85 clone containing the same sequence was found at disease relapse 100 weeks post allo-HSCT. Using quantitative real-time PCR $[23,24]$, we found that the expression level of the malignant $\mathrm{V} \delta 5+\mathrm{T}$ cell clone was high at diagnosis and low when disease relapse was detected. In addition, the V85 expression level declined even further after chemotherapy at 108 weeks when the disease achieved remission with minimal residual disease (i.e., $4 \%$ blast cells in the bone marrow) (Figure 3). These data further indicate the significance of dynamically monitoring malignant $\mathrm{T}$ cell clones to molecularly identify relapse, which may provide evidence for commencing treatments such as preventive anti-leukemia therapy to clinically inhibit disease relapse.

The identification of malignant T-ALL cell clones was performed at different times using Southern blot, PCR, RT-PCR, GeneScan, FT-CGH, and next generation sequencing spectratyping [25]. The distribution profiles and clonality of the TCR repertoire in T cells could be characterized using RT-PCR and GeneScan [17]. While an advantage of the FT-CGH and LM-PCR techniques is that they can identify chromosomal breakpoints and unique, high percentage $\mathrm{T}$ cell clones in a sample, they cannot characterize the polyclonal TCR subfamily distribution or a small fraction of $\mathrm{T}$ cell clones [12]. The novel, exhaustive $T$ cell repertoire sequencing technique can directly measure the TCR repertoire size of at least 1 million clonotypes [25]. Therefore, the combination of the FT-CGH, LM-PCR, PCR, and GeneScan techniques for characterizing $\mathrm{T}$ cell malignancies is an ideal serial method not only for identifying abnormal chromosome rearrangements in malignant $\mathrm{T}$ cell clones but also for detecting the evolution of malignant $\mathrm{T}$ cell clones for the diagnosis, prognosis, and evaluation of reactive $\mathrm{T}$ cell clones to characterize the immune status of patients and develop specific immunotherapies.

\section{Clonally expanded reactive $\mathrm{T}$ cell clone}

Numerous studies have demonstrated that specific clonally expanded $\mathrm{T}$ cells may be identified in the PB of patients with cancer and leukemia, demonstrating their specific anti-leukemic cytotoxicity in vitro [13,26-28]. Moreover, clonally expanded $\mathrm{T}$ cells may be derived from donor lymphocytes after allo-HSCT or donor lymphocyte infusion (DLI), which may increase survival following alloHSCT in patients with advanced-stage acute leukemia $[26,29]$. These clonally expanded T cells are derived from TCR $\alpha \beta$ or $\gamma \delta$ T cells [10]. Increasing data have demonstrated that $\gamma \delta$ T cells may be used to develop specific immunotherapies for patients with cancers such as bladder cancer and hepatocellular carcinoma [30]. After transplantation, the patient achieved $\mathrm{CR}$, and the distribution and clonality of the TCR $\mathrm{V} \gamma$ and $\mathrm{V} \delta$ subfamilies were examined. While a malignant $\mathrm{V} \delta 5+\mathrm{T}$ cell clone was not detected, oligoclonally expanded V $\delta 4$ subfamily $\mathrm{T}$ cells were identified in samples from all time points (i.e., 4, 8, 68,100 , and 108 weeks post allo-HSCT) even after the patient underwent relapse (100 weeks post allo-HSCT; Figures $2 \mathrm{C}$ to $\mathrm{G}$ ). The TCR sequence of the $\mathrm{V} \delta 4+\mathrm{T}$ cell clone was identified as V V4D $\delta 3 \mathrm{~J} \delta 1$ by direct sequencing, and the same TCR rearrangement was confirmed in all samples from each of the time points post transplantation (Figure 4). The V $\delta 4+\mathrm{T}$ cell clone increased more than three-fold when the patient reached CR status post alloHSCT. Interestingly, the V $\delta 4$ expression level gradually increased 100 and 108 weeks post allo-HSCT, which was when disease relapse occurred and after chemotherapy was given, respectively (Figure 5). This observation suggests that the increased $\mathrm{V} \delta 4+\mathrm{T}$ cell clone may be a reactively expanded $\mathrm{T}$ cell clone that has specific anti-T-ALL function. Although the patient underwent GVHD 8 weeks post transplantation, which was subsequently controlled, the continuously expanded $\mathrm{T}$ cell clone may be not related to GVHD. The higher proportion of V $84+\mathrm{T}$ cell clones in samples taken after disease relapse may further support the idea of its role as a reactively expanded anti-leukemia T-cell clone. These results are similar to findings by Meeh et al. who demonstrated that $\mathrm{V} \delta 1+\mathrm{T}$ cells respond to acute leukemia [28]. However, further characterization of the biological functions of the $\mathrm{V} \delta 4+\mathrm{T}$ cell clone is needed; the reactive $\mathrm{T}$ cell clone may be amplified and used to study adoptive anti-leukemia immunotherapy, moreover, the TCR V $\delta 4$ and its pattner $\mathrm{V} \gamma$ gene could be used for transfer and the modification of normal $\mathrm{T}$ cells for identification their anti-leukemia effect $[6,14,31,32]$.

In summary, the evolution of malignant TCR $\gamma \delta+$ and reactive $\mathrm{T}$ cell clones was identified in a patient with relapse T-ALL before and after allo-HSCT and at relapse post alloHSCT. The techniques used in this study establish the sensitive detection of malignant and reactive $T$ cell clones, and the identified $\mathrm{T}$ cell clones may serve not only as biomarkers for minimal residual disease detection but also as anti-leukemia immune status indicators in patients who achieved CR.

\section{Additional file}

\section{Additional file 1: Table S1. Details of Clinical therapy for the patient with relapse T-ALL. Table S2. Clinical patient characteristics. Table S3. List of primers used for the V $\delta 5$ TCR PCR.}

\section{Competing interests}

The authors declare that they have no competing interests.

\section{Authors' contributions}

YQL contributed to the concept development and study design. SHC performed the RT-PCR, GeneScan and real-time PCR analyses, HTZ performed LM-PCR, SXG prepared the RNA and CDNA, XLW and LY prepared the PBMCs and DNA, and $\mathrm{XH}, \mathrm{JYW}$ and $\mathrm{XD}$ were responsible for treatment of the patient and performed clinical data acquisition. YQL, SHC and XH coordinated the study and helped draft the manuscript. All authors read and approved the final manuscript. 


\section{Acknowledgements}

This work was supported by grants from the National Natural Science Foundation of China (No. 30871091, 91129720 and 81270604), the Collaborated grant for HK-Macao-TW of the Ministry of Science and Technology (2012DFH30060), the Fundamental Research Funds for the Central Universities (No. 21610603, 21612116) and the Guangdong Science \& Technology Project (No. 2012B050600023).

\section{Author details}

${ }^{1}$ Key Laboratory for Regenerative Medicine of Ministry of Education, Jinan University, Guangzhou 510632, China. ${ }^{2}$ Institute of Hematology, Medical College, Jinan University, Guangzhou 510632, China. ${ }^{3}$ Department of Hematology, Guangdong General Hospital (Guangdong Academy of Medical Sciences), Guangzhou 510080, China.

Received: 20 April 2013 Accepted: 10 July 2013

Published: 12 July 2013

\section{References}

1. Kraszeska MD, Dawidowska M, Szczepański T, Witt M: T-cell acute lymphoblastic leukemia: recent molecular biology findings. $\mathrm{Br} J$ Haematol 2012, 15:303-315.

2. Cleaver AL, Beesley AH, Firth MJ, Sturges NC, O'Leary RA, Hunger SP Baker DL, Kees UR: Gene-based outcome prediction in multiple cohorts of pediatric T-cell acute lymphoblastic leukemia: a Children's Oncology Group study. Mol Cancer 2010, 9:105.

3. Aifantis I, Raetz E, Buonamici S: Molecular pathogenesis of T-cell leukaemia and lymphoma. Nat Rev Immunol 2008, 8:380-390.

4. Wang Y, Liu DH, Xu LP, Liu KY, Chen H, Chen YH, Han W, Zhang XH, Huang XJ: Haploidentical/mismatched hematopoietic stem cell transplantation without in vitro T cell depletion for T cell acute lymphoblastic leukemia. Biol Blood Marrow Transplant 2012, 18:716-721.

5. LV M, Huang X: Allogeneic hematopoietic stem cell transplantation in China: where we are and where to go. J Hematol Oncol 2012, 5:10.

6. Rezvany MR, Jeddi-Tehrani M, Wigzell $\mathrm{H}$, Osterborg A, Mellstedt $\mathrm{H}$ : Leukemia-associated monoclonal and oligoclonal TCR-BV use in patients with B-cell chronic lymphocytic leukemia. Blood 2003, 101:1063-1070.

7. De Rijke B, Fredrix H, Zoetbrood A, Scherpen F, Witteveen H, De Witte T, van de Wiel-Van Kemenade E, Dolstra H: Generation of autologous cytotoxic and helper T-cell responses against the B-cell leukemia-associated antigen HB-1: relevance for precursor B-ALL-specific immunotherapy. Blood 2003, 102:2885-2891.

8. Zheng H, Chen Y, Chen S, Niu Y, Yang L, Li B, Lu Y, Geng S, Du X, Li Y: Expression and distribution of the PPP2R5C gene in leukemia. J Hematol Oncol 2011, 4:21

9. Huang $X$, Du X, Li Y: The role of BCL11B in hematological malignancy. Exp Hematol Oncol 2012, 1:22.

10. Bellone M, Svensson AM, Zaslav AL, Spitzer S, Golightly M, Celiker M, Hu Y, Ma Y, Ahmed T: Pediatric T-cell prolymphocytic leukemia with an isolated 12(p13) deletion and aberrant CD117 expression. Exp Hematol Oncol 2012, 1:7.

11. Langerak AW, van Den Beemd R, Wolvers-Tettero IL, Boor PP, Van Lochem EG, Hooijkaas H, Van Dongen JJ: Molecular and flow cytometric analysis of the $\mathrm{V} \beta$ repertoire for clonality assessment in mature TCRaßT-cell proliferations. Blood 2001, 98:165-173.

12. Przybylski GK, Dik WA, Wanzeck J, Grabarczyk P, Majunke S, Martin-Subero II, Siebert R, Dölken G, Ludwig WD, Verhaaf B, Van Dongen JJ, Schmidt CA, Langerak AW: Disruption of the BCL11B gene through inv(14) (q11.2q32.31) results in the expression of BCL11B-TRDC fusion transcripts and is associated with the absence of wild-type $B C L 11 B$ transcripts in T-ALL. Leukemia 2005, 19:201-208.

13. Tanaka-Harada Y, Kawakami M, Oka Y, Tsuboi A, Katagiri T, Elisseeva OA, Nishida S, Shirakata T, Hosen N, Fujiki F, Murao A, Nakajima H, Oji Y, Kanda $Y$, Kawase I, Sugiyama $\mathrm{H}$ : Biased usage of BV gene families of T-cell receptors of WT1 (Wilms' tumor gene)-specific CD8+ T cells in patients with myeloid malignancies. Cancer Sci 2010, 101:594-600.

14. Wang Q, Liu H, Zhang X, Liu Q, Xing Y, Zhou X, Tong C, Zhu P: High doses of mother's lymphocyte infusion to treat EBV-positive T-cell lymphoproliferative disorders in childhood. Blood 2010, 116:5941-5947.

15. Przybylski GK, Dittmann K, Grabarczyk P, Dölken G, Gesk S, Harder L, Landmann E, Siebert R, Schmidt CA: Molecular characterization of a novel chromosomal translocation $\mathrm{t}(12 ; 14)(\mathrm{q} 23 ; \mathrm{q} 11.2)$ in T-lymphoblastic lymphoma between the T-cell receptor delta-deleting elements (TRDREC and TRAJ61) and the hypothetical gene C12orf42. Eur J Haematol 2010, 85:452-456.

16. Li Y, Chen S, Yang L, Li B, Chan JYH, Cai D: TRGV and TRDV repertoire distribution and clonality of T cells from umbilical cord blood. Transpl Immunol 2009, 20:155-162.

17. Puisieux I, Even J, Pannetier C, Jotereau F, Favrot M, Kourilsky P: Oligoclonality of tumor-infiltrating lymphocytes from human melanomas. J Immunol 1994, 153:2807-2818.

18. Weng JY, Du X, Geng SX, Peng YW, Wang Z, Lu ZS, Wu SJ, Luo CW, Guo R, Ling W, Deng CX, Liao PJ, Xiang AP: Mesenchymal stem cell as salvage treatment for refractory chronic GVHD. Bone Marrow Transplant 2010, 45:1732-1740.

19. Vanura K, Vrsalovic MM, Le T, Marculescu R, Kusec R, Jäger U, Nadel B: V(D)」 targeting mistakes occur at low frequency in acute lymphoblastic leukemia. Genes Chromosomes Cancer 2009, 48:725-736.

20. Thulien KJ, Belch AR, Reiman T, Pilarski LM: In non-transplant patients with multiple myeloma, the pre-treatment level of clonotypic cells predicts event-free survival. Mol Cancer 2012, 11:78.

21. Kode J, Advani S, Chiplunkar S: T-cell receptor gamma and delta gene rearrangements in T-cell acute lymphoblastic leukemia in Indian patients. Leuk Lymphoma 2000, 36:331-338.

22. Langerak AW, Wolvers-Tettero IL, van den Beemd MW, Van Wering ER, Ludwig WD, Hählen K, Necker A, Dongen JJ: Immunophenotypic and immunogenotypic characteristics of TCR $\delta \delta+T$ cell acute lymphoblastic leukemia. Leukemia 1999, 13:206-214

23. Stams WAG, Den Boer ML, Beverloo HB, Meijerink JPP, Stigter RL Van Wering ER, Janka-Schaub GE, Slater R, Pieters R: Sensitivity to $\mathrm{L}$-asparaginase is not associated with expression levels of asparagine synthetase in $\mathrm{t}(12 ; 21)^{+}$pediatric ALL. Blood 2003, 101:2743-2747.

24. Chen S, Zha X, Yang L, Li B, Zhong L, Li Y: Deficiency of CD $3 Y, \delta$, $\varepsilon$ and $Z$ expression in T-cells from AML patients. Hematology 2011, 16:31-36.

25. Warren RL, Freeman JD, Zeng T, Choe G, Munro S, Moore R, Webb JR, Holt RA: Exhaustive T-cell repertoire sequencing of human peripheral blood samples reveals signatures of antigen selection and a directly measured repertoire size of at least 1 million clonotypes. Genome Res 2011, 21:790-797.

26. Zha X, Chen S, Yang L, Li B, Chen Y, Yan X, Li Y: Characterization of the CDR3 structure of V $321 \mathrm{~T}$ cell clone in patients with P210 ${ }^{B C R-A B L}$-positive CML and B-ALL. Hum Immunol 2011, 72:798-804.

27. Yin Q, Zha X, Yang L, Chen S, Zhou Y, Wu X, Li Y: Generation of diffuse large $B$ cell lymphoma-associated antigen-specific Va6/V $\beta 13$ + T cells by TCR gene transfer. J Hematol Oncol 2011, 4:2.

28. Meeh PF, King M, O'Brien RL, Muga S, Buchhalts P, Meuberg R, Lamb LS Jr: Characterization of the $\gamma \delta$ T cell response to acute leukemia. Cancer Immunol Immunother 2006, 55:1072-1080.

29. Wang Y, Liu DH, Fan ZP, Sun J, Wu XJ, Ma X, Xu LP, Liu KY, Liu QF, Wu DP, Huang $X J$ : Prevention of relapse using DLI can increase survival following HLA-identical transplantation in patients with advanced-stage acute leukemia: a multi-center study. Clin Transplant 2012, 26:635-643.

30. Cabillic F, Toutirais $O$, Lavoué $V$, de La Pintière $C T$, Daniel P, Rioux-Leclerc N, Turlin B, Mönkkönen H, Mönkkönen J, Boudjema K, Catros V, Bouet-Toussaint F: Aminobisphosphonate-pretreated dendritic cells trigger successful Vgamma9Vdelta2 T cell amplification for immunotherapy in advanced cancer patients. Cancer Immunol Immunother 2010, 59:1611-1619.

31. Li Y, Lin C, Schmidt CA: New insights into antigen specific immunotherapy for chronic myeloid leukemia. Cancer Cell Int 2012, 12:52.

32. Kessels HW, Wolkers MC, van den Boom MD, van der Valk MA, Schumacher TN: Immunotherapy through TCR gene transfer. Nat Immunol 2001, 2:957-961.

doi:10.1186/1476-4598-12-73

Cite this article as: Chen et al:: The evolution of malignant and reactive $\gamma \delta+T$ cell clones in a relapse T-ALL case after allogeneic stem cell transplantation. Molecular Cancer 2013 12:73. 